\title{
A Closed-Form Solution of Photon States in Generic Gravitational Fields
}

\author{
Xiaoping $\mathrm{Hu}$ \\ Boly Inc. \\ 3235 Kifer Rd., Suite 260, Santa Clara, CA 95051. \\ Email: Xiaoping_Hu@bolymedia.com \\ ORCID: 0000-0001-8026-6965
}

\begin{abstract}
This article first examines the solutions of two special cases of photon states (position, velocity, and frequency), which prove that the isotropy assumption underlies general relativity is invalid. As such, the geodesic methods used in general relativity cannot be used in general, though it may work in special cases. Then a method based on gravitational decomposition is proposed that solves the photon states in any gravitational fields in closed form. The solution does not even involve time $\mathrm{t}$ because time dilation is automatically covered in the decomposition solutions by a priori, proven solutions.
\end{abstract}

Key Words: Photon, Gravitaional Field, Special Relativity, General Relativity, Equivalence Principle, Geodesic Principle, Energy Conservation Principle, Isotropy, Gravitational Redshift, Shwarzchild Sphere, Photon Sphere, Great Photon Circle, Black Hole.

\section{Introduction}

General Relativity has been regarded a major science break-through in the past 100 years. It has made a lot of amazing predictions and has been tested by numerous observations and experiments. Therefore, to challenge its foundation is an extraordinary claim and extraordinary claim requires extraordinary discoveries. This paper first examines the solutions of two special cases of photon states, which can be re-evaluated based on symmetry consideration and energy conservation principle ${ }^{1-6}$, while fully complying with the special relativity principle ${ }^{1-6}$. The solutions of these two special cases (consistent with general relativity), which are non-disputable in form and have been mostly proven in the past, prove that the reaction of photon to gravitational field is non-isotropic if special relativity principle holds. As such, conventional vector mathematics (that assumes symmetry and isotropy) breaks down. The generic general relativity theory assumes symmetry and isotropy. Now that isotropy is broken, the current computational methods in general relativity cannot be used in general.

To cope with the non-isotropic issue, a new method is proposed that fully complies with the Energy Conservation Principle and the Special Relativity Principle, but without assuming isotropy. As a result, the new but much simpler equations obtained do not involve time $t$ and solves the photon states in 3D space in closed form. The time dilation issue is automatically satisfied in the process because each of the decomposed solutions satisfies Special Relativity Principle.

2. Special Case I: Photon travels along gravitational line 
In this section, we first examine the solution of a special case, in which the photon's velocity is parallel to the gravitational lines. In this article, we shall use bold faced letters to denote vector, and regular letters to denote scalars. To make it simple and concrete, let's consider the case of a black hole and a photon travels along a radial line toward the center of the black hole, as illustrated in Fig. 1, in which $\mathrm{P}_{1}$ is a photon travelling along a radial line $\mathbf{r}$ toward the center of a black hole with mass $\mathrm{M}$, such that the velocity $\mathbf{V}$ of $\mathrm{P}_{1}$ is parallel to $\mathbf{r}$, or $\mathbf{r} \times \mathbf{V}=\mathbf{0}$.

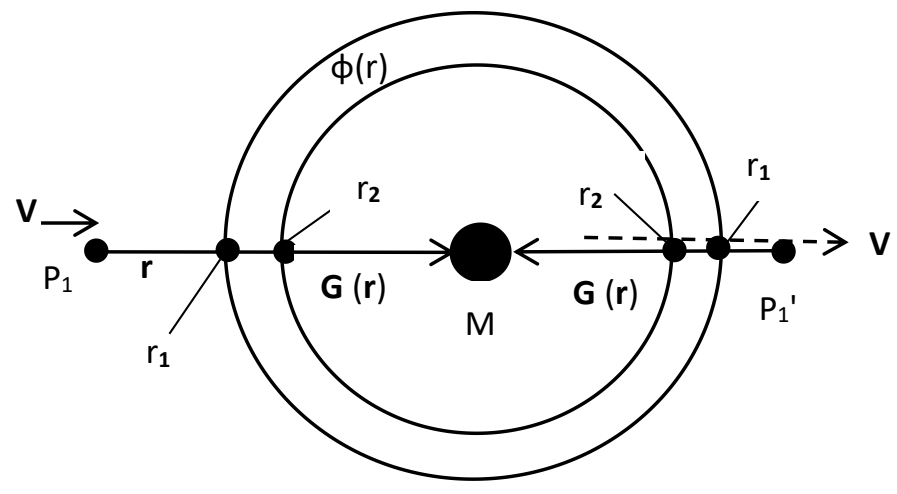

Fig. 1. Photon travels along gravitational line

This is a well studied case, so we'll first use the results from the textbooks ${ }^{1-3}$. The black hole with mass $M$ would generate a gravitational field $\mathbf{G}(\mathbf{r})$, which would generate a potential field $\phi(r)$ as follows:

$$
\begin{aligned}
& \mathbf{G}(\mathbf{r})=\frac{G_{N} M}{r^{2}} \mathbf{n}_{\mathbf{r}} \\
& \phi(\mathbf{r})=\frac{G_{N} M}{r}
\end{aligned}
$$

where $\mathbf{n}_{\mathbf{r}}$ is a unit vector along radius vector $\mathbf{r}$, and $\mathbf{r}=\|\mathbf{r}\|$ is the length of the radius vector $\mathbf{r}$. With the well-established result, the velocity of $\mathrm{P}_{1}$ will not be changed (due to special relativity principle), but the frequency $f$ of $\mathrm{P}_{1}$ will be changed (due to time dilation) according to the following formula:

$$
\begin{aligned}
& \Delta \mathrm{f} / \mathrm{f}=\Delta \phi(\mathbf{r}) / \mathrm{c}^{2}=\frac{G_{N} M}{r^{2} c^{2}} \Delta \mathrm{r}, \text { or } \\
& \Delta \mathrm{f} / \mathrm{f}=(\mathbf{G}(\mathbf{r}) \bullet \Delta \mathbf{r}) / \mathrm{c}^{2}
\end{aligned}
$$

where $\mathrm{c}$ is the speed of light, and $\Delta$ a differential operator. Note that Eq. (4) holds for any $\mathbf{G ( r )}$ as long as $G(r)$ is parallel to the velocity of the photon.

All the equations above are consistent with existing General Relativity theory.

When photon travels along the radial line, in the space-time domain, it travels along a line that bifurcating the angle between axis $r$ and ct equally ${ }^{1-3}$ with $r=c t($ or $r=-c t)$. This basically shows that the speed of the photon on the radial line is constantly c. So, the space-time methodology does not bring in any new information compared to the 3D situation. When looking in the 3D space, because time dilation has already been taken care of in $\Delta \mathrm{f} / \mathrm{f}$ and the photon has constant speed along $\mathbf{r}$, Eq. (4) holds for every $\mathbf{r}$, which does not involve time $t$. 
Now we shall revisit the concept of "Gravitational Mass". Since photon has been regarded a massless particle, in the past, Gravitational Mass of photon has been constantly been avoided in general relativity studies ${ }^{1-3}$. However, we shall show in this article that this concept is very useful and can produce the correct results. The Gravitational Mass $\mathrm{m}_{\mathrm{g}}$ of a photon is given by

$$
\mathrm{m}_{\mathrm{g}}=\mathrm{hf} / \mathrm{c}^{2}
$$

where $\mathrm{h}$ is Planck constant. Gravitational Mass is considered the same of the photon at rest. Now, if we assume $\mathrm{P}_{1}$ is a particle with mass $\mathrm{m}_{\mathrm{g}}$ as in Eq. (5) and use the Newtonian gravitational method and the Energy Conservation Principle, we would have

$$
\left(\mathbf{G}(\mathbf{r}) \mathrm{m}_{\mathrm{g}} \bullet \Delta \mathbf{r}\right)=\Delta \mathrm{W}=\mathrm{h} \Delta \mathrm{f}
$$

where $\left(\mathbf{G}(\mathbf{r}) \mathrm{m}_{\mathrm{g}}\right)$ is the Newtonian gravitational force of $\mathbf{G}(\mathbf{r})$ exerted on photon, $\Delta \mathrm{W}$ the work that gravitational force $\left(\mathbf{G}(\mathbf{r}) \mathrm{m}_{\mathrm{g}}\right)$ does in distance span $\Delta \mathbf{r}$, and $\mathrm{h} \Delta \mathrm{f}$ the energy increase of the photon obtained because of the work $\Delta \mathrm{W}$ done by $\mathbf{G}(\mathbf{r}) \mathrm{m}_{\mathrm{g}}$. By replacing Eq. (5) into Eq. (6), we would get Eq. (4). That means, when the gravitational field is parallel to the velocity of photon, by assuming all the work the gravitational field done to the photon is converted to the energy increase of the photon, we can treat the photon as having a Gravitational Mass in a Newtonian gravitational field.

Because the energy increase of the photon absorbs all the work of the gravitational force, therefore, the photon will not have any acceleration, and hence we don't have to worry about time dilation. That means, there is no time dilation because the work a parallel gravitational field has done is converted to the increase of the energy/frequency (or gravitational mass) of the photon.

Although, the results in this section does not necessarily need the concept of Gravitational Mass. We shall show in the next section, Gravitational Mass is a very useful concept.

To summarize above, we can have the following Postulate:

Postulate 1. When gravitational field is parallel to the velocity of a photon, both the speed and direction of the photon will not change, but only the frequency will change according to Eq. (4).

In the above we see two methodological differences in viewing the photon's behaviour in gravitational field. The first one is the space-time method in the traditional general relativity theory, in which gravitational field will cause time dilation and photon's states has to be computed in space-time. The other view presented in this article is that when the gravitational field is parallel to the velocity of the photon, because there is no change of the speed, hence no acceleration and no time dilation is involved. The photon balances the influence of gravitational field by changing its frequency. In this second view, we can treat photon as a particle with gravitational mass and solves its states in $3 \mathrm{D}$ space.

Now let's do more study on Eq. (4). Eq, (4) is obtained from both the space-time method and the gravitational mass method and is valid for any $\mathbf{r}$. Since $\mathbf{G}(\mathbf{r})$ is parallel to $\Delta \mathbf{r}$, replacing $\Delta$ by the other differential operator d, the Eq. (4) gives

$$
\mathrm{df} / \mathrm{f}=\|\mathbf{G}(\mathbf{r})\| \mathrm{dr} / \mathrm{c}^{2}
$$

where $\|\mathbf{G}(\mathbf{r})\|$ is the norm of $\mathbf{G}(\mathbf{r})$. Now, replacing Eq. (1) into Eq. (7), we would get

$$
\mathrm{df} / \mathrm{f}=\left(\mathrm{G}_{\mathrm{N}} \mathrm{M} / \mathrm{c}^{2}\right)\left(\mathrm{dr} / \mathrm{r}^{2}\right)
$$


Regardless of whether the photon takes a space-time path (as suggested from a view point of general relativity) or a radial path (as suggested by the view of gravitational mass), Eq. (8) is valid for every $r$. Therefore, we can integrate the right side of Eq. (8) over $r$ and left side over $f$. Integrating both sides of Eq. (8) gives

$$
\ln \left(\mathrm{f}_{2} / \mathrm{f}_{1}\right)=\left(\mathrm{G}_{\mathrm{N}} \mathrm{M} / \mathrm{c}^{2}\right) *\left(1 / \mathrm{r}_{2}-1 / \mathrm{r}_{1}\right)
$$

or

$$
f_{2}=f_{1} * \exp \left(\left(G_{N} M / c^{2}\right) *\left(1 / r_{2}-1 / r_{1}\right)\right)
$$

where $r_{1}$ and $r_{2}$ are two positions of photon $P_{1}$ on $\mathbf{r}$, and $f_{1}$ and $f_{2}$ are the frequencies of photon $P_{1}$ on these two positions.

Now, since in the above example, $\mathrm{M}$ is the mass of a black hole with very large value, when photon reaches the center of the black hole, $\mathrm{r}_{2}$ is close to zero. Therefore, irrespective of the initial frequency $f_{1}$ of the photon, when it reaches the center of a black hole, its frequency will reach almost infinity, meaning that it will become a gamma ray. Therefore, we have the following Postulate:

Postulate 2. Any light, irrespective of its original frequency, when it reaches the center of a black hole, it will become a gamma ray.

Postulate 2 explains the gamma ray emission near black holes that has puzzled astrophysicists till this date, because any light will become gamma ray when it approaches the center of a black hole. Now, Fig. 1 has shown a case where $\mathrm{P}_{1}$ is moving toward the center of a black hole. Then what happens when it is moving away from the center of a black hole? As long as the black hole's temperature is not absolute zero, it also has black body emissions.

Now consider Photon $\mathrm{P}_{1}$ ' leaving the black hole along radial (gravitational lines) in Fig. 1. From Fig. 1 and Eq. (6), The frequency change equation of Eq. (10) now becomes

$$
f_{1}=f_{2} * \exp \left(\left(G_{N} M / c^{2}\right) *\left(1 / r_{1}-1 / r_{2}\right)\right)
$$

where $f_{1}$ is the frequency of $P_{1}$ ' on radius $r_{2}$ and $f_{2}$ is the frequency of $P_{1}$ ' on radius $r_{2}$, such that when $r_{1}$ increases, $f_{1}$ reduces. Contrary to the well established understanding that a photon inside the event horizon of a black hole ${ }^{1-3}$ can never escape it, the black hole cannot change the speed of the photon leaving it along radial lines and hence cannot pull it back, as can be seen from the situation of photon $\mathrm{P}_{1}{ }^{\prime}$ in Fig. 1. All a black hole can do is to reduce the photon's frequency when it moves away from the black hole as long as it is moving along the gravitational line. That means the gamma rays that penetrated the black hole can still escape the black hole, and gamma rays has excellent penetration capability. Therefore, if any ray can pass through the black hole, its frequency will still be very high near the black hole. And because the velocity of photon is not changed, yes, those gamma rays that run parallel to the radial gravitational lines of a black hole can indeed escape! That's a surprise.

Any none-gamma ray leaving a black hole is likely to become a microwave ray due to the dramatic frequency reduction by the black hole. This offers another explanation to the Cosmological Microwave Background (CMB) radiation of the Universe.

\section{Special Case II: Photon runs on a perfect circle}

While in reality, perfect things rarely exists, here, a perfect circle just makes discussion 
simple. Our results do not depend on it. In what situations a photon will keep running on a perfect circle? One immediately can think of Schwarzchild Sphere ${ }^{1-3}$ or Photon Sphere ${ }^{1}$. However, as we shall show in this section, the results of Schwarzchild Sphere and Photon Sphere are both derived under the Equivalence Principle and Geodesic Principle, which may not be valid for photons in general. Therefore, we shall consider a more generic case and discuss Schwarzchild Sphere and Photon Sphere as special cases.

Fig. 2 shows a gravitational field with circular symmetry. Assume that photon $\mathrm{P}_{2}$ can find a radius $\mathrm{R}$ so that $\mathrm{P}_{2}$ can keep running on the circle while achieving balance with the gravitational field $\mathbf{G}(\mathbf{r})$, which is circularly symmetric. We shall call such a circle as photon's Great Photon Circle (on a Photon Sphere). A Great Photon Circle is a largest circle on a Photon Sphere, on which the gravity influence to photon is somehow balanced. In the theory of general relativity, this balance effect is due to the bending of space-time. However, we should not take this as the ground truth.

First, assume the Schwarzhchild Sphere ${ }^{1-3}$ derived from General Relativity is correct. Then, in Fig. 2, if $\mathrm{M}$ is the mass of a black hole, and when the Radius R becomes the Schwarzchild Radius $\mathrm{R}_{\mathrm{S}}$ that satisfies

$$
\mathrm{R}_{\mathrm{S}}=2 \mathrm{G}_{\mathrm{N}} \mathrm{M} / \mathrm{c}^{2}
$$

Then, according to general relativity, photon $\mathrm{P}_{2}$ can run on any big circle on the Schwarzhchild Sphere for ever because Schwarzhchild Sphere is a geodesic sphere.

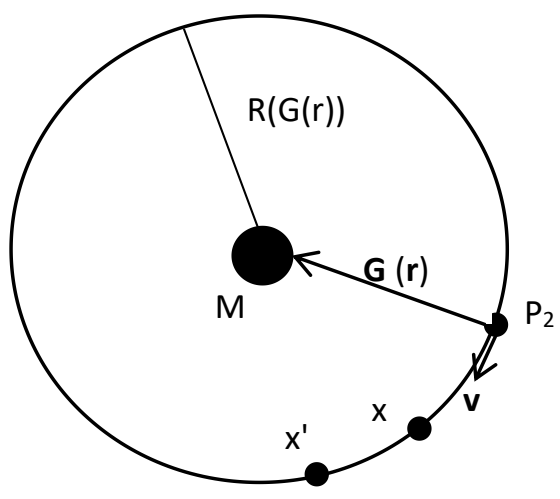

Fig. 2. Photon travels in a perfect circle

It has been understood ${ }^{1-3,7}$ that the Schwarzhchild Sphere represents an event horizon that inside it, no photons can escape. But what about on the Schwarzhchild Sphere? Nothing has been said on photons running on the Schwarzhchild Sphere. If any photon inside Schwarzhchild Sphere will be absorbed into the black hole, and any photon outside of the Schwarzhchild Sphere can escape, then it's possible that a photon on the Schwarzhchild Sphere can stay there. So we'll temporarily treat any great circle on a Schwarzhchild Sphere as a potential Great Photon Circle.

Another sphere derived from general relativity is Photon Sphere ${ }^{1-3}$, on which photons will keep running for ever. The radius of Photon Sphere is given as

$$
\mathrm{R}_{\mathrm{P}}=3 \mathrm{G}_{\mathrm{N}} \mathrm{M} / \mathrm{c}^{2}
$$

Therefore, any great circle on Photon Sphere is definitely a candidate for Great Photon Circle.

So from the above, suffice it to say that for an circularly symmetric gravitational field of a 
black hole, there exist a Great Photon Circle, on which, a photon $\mathrm{P}_{2}$ can run on it for ever. When $\mathrm{P}_{2}$ runs on such a circle, it has to satisfy the following conditions:

A. its speed is constantly c (by Special Relativity Principle);

B. its frequency will never change (by Energy Conservation Principle).

Once the radius $\mathrm{R}(\mathbf{G}(\mathbf{r}))$ of the Great Photon Circle is know, then, if we know the position $\mathbf{x}$ of $\mathrm{P}_{2}$ at $t$, it is then straight forward to derive the position $\mathbf{x}^{\prime}$ at $\mathrm{t}^{\prime}$, as shown in Fig. 2, because the speed of $\mathrm{P}_{2}$ on the Great Photon Circle is always c.

Because mass and force have been intentionally avoided in general relativity, we now add another explanation for Condition B from symmetry: because any point on the circle is circularly symmetric to another, if the frequency of $\mathrm{P}_{2}$ keeps changing (increasing or reducing), it will keep changing for ever. And this is impossible, therefore Condition B above must be satisfied. The above discussion leads to the following Postulate:

Postulate 3. A photon on its Great Photon Circle will have constant frequency and constant velocity, but the direction of the velocity keeps changing and is perpendicular to the radius of the Great Photon Circle at any point.

That means, when a photon is running on its Great Photon Circle, the gravitational field has no impact on its frequency and speed, but can only change its direction of velocity. Conceptually, on the Great Photon Circle, the photon balances the impact of gravitational field by centrifugal tendency.

Now, Postulate 1 and Postulate 3 clearly prove that the impact of gravitational field on a photon does not have the isotropic property, which underlies the Equivalence Principle and Geodesic Principle. Therefore, we contend that the Equivalence Principle and Geodesic Principle in general relativity do not hold in general, because in previous researches, no special attention is paid to the angle between the velocity of the photon and the gravitational field, which keeps changing from place to place. As a matter of fact, in most previous researches ${ }^{1-3}$, gravitation field function $\phi$ is used, which is a scalar function, not even a vector function.

Now that both Schwarzhchild Sphere and Photon Sphere could be wrong candidates for Great Photon Circle of a photon, then, does such circle exist for a given circularly symmetric gravitation field? If so, what is it?

Since a gravitational field perpendicular to photon's velocity can indeed make the light path bending, then for any circularly symmetric gravitational field, a Great Photon Circle exists (otherwise the bending will be arbitrary). We shall now present a solution of such a circle using the concept of Gravitational Mass. Even if such a solution is disproved by experiments, the existence of a Great Photon Circleshould not be doubted due to symmetric considerations and the fact that gravitational field can indeed bend light.

In section 2 we have shown that, correct results have been obtained by assuming photon has a gravitational mass. Because mass is a directionless property, therefore, it is reasonable to think that when the gravitational force is not parallel to the velocity of the photon, the mass is still the same. Therefore, we can use the balance of the Newtonian gravitational force and the centrifugal 
force of the photon to determine the radius of the Great Photon Circle as follows:

$$
\mathrm{G}\left(\mathrm{R}_{\mathrm{B}}\right) * \mathrm{~m}_{\mathrm{g}}=\mathrm{m}_{\mathrm{g}} \mathrm{c}^{2} / \mathrm{R}_{\mathrm{B}}
$$

and we can get

$$
R_{B}=c^{2} / G\left(R_{B}\right)
$$

where $R_{B}$ is the radius of the Great Photon Circle, $G\left(R_{B}\right)$ is the strength of the gravitational field on the Great Photon Circle, $\mathrm{m}_{\mathrm{g}}$ the gravitational mass of the photon. Now Eq. (14) shows that the radius $\mathrm{R}_{\mathrm{B}}$ of the Great Photon Circle is not relevant to the gravitational mass of the photon, as long as it is not zero. That means, Eq. (14) could in fact be correct.

For a black hole with Newtonian gravitational field, $G\left(R_{B}\right)=G_{N} M / R^{2}$, then $R_{B}=R_{S} / 2$. Eq. (14) is more generic, because it takes any, generalized gravitational field, though the correctness of it requires future experimental proof.

The correctness of Eq. (14) can be verified or improved by experiments. Qualitatively, when $G\left(R_{B}\right)$ is $0, R_{B}$ is infinity, indicating no bending on photon's trajectory, and when $G\left(R_{B}\right)$ is very large, $R_{B}$ becomes small, indicating strong bending. So, Eq. (14) meets our intuitive expectation.

In the above, we have three candidates for Great Photon Circle, though we do not know which one is correct at this point. Suffice it to say that for any circularly symmetric gravitational field of any strength, a Great Photon Circle exists, on which, photon balances the effect of gravitational field by directional change. If Eq. (14) is proven by experiments, then the concept of Gravitational Mass becomes valid.

While Special Relativity forbids photon to have mass, again, we should not take this as the ground truth. Assuming photon has mass does not affect the major results of this paper. However, if in the future, Eq. (14) is proven by experiments or observation, then, a new fact about photon is established.

The reason we use letter $B$ to denote the radius of Great Photon Circle is because $R_{B}$ is actually the radius of the bending curvature of photon responding to a gravitational field $\mathrm{G}$ perpendicular to the velocity of the photon, due to the following Postulation:

Postulate 4. At each localized point, a gravitational field perpendicular to the velocity of a photon cannot change its frequency and speed, but only change its direction of velocity, as long as the photon changes its direction as if it is running on a Great Photon Circle defined by the localized gravitational field.

Postulation 4 is a generalized form of Postulate 3 and similar method has been used in many differential geometric computations. We shall call $\mathrm{R}_{\mathrm{B}}$ the Bending Radius.

In the above Postulation, how the Great Photon Circle is obtained is irrelevant. What matters is that if photon runs on the Great Photon Circle defined by the local value of the perpendicular component of a gravitational field, then Special Relativity Principle is satisfied, and the frequency of the photon is not changed by the perpendicular component of the gravitational field.

That means, though the Great Photon Circle is the end result of the trajectory of a photon in the 3D space, this 3D space result automatically satisfies the Special Relativity Principle and Energy Conservation Principle. Therefore, there is no need to invoke space-time to compute the trajectory of the photon. 


\section{A closed-form solution of photon states in a generic gravitational field}

Though elegant mathematics has been developed for general relativity and some impressive predictions have been obtained ${ }^{1-3}$, the space-time mathematics involves a lot of tensors in 4 dimensions, and hence requires a lot of computation and can easily lead to mistakes. Not only so, as Section 3 has shown, the isotropy property of photon's reaction to a gravitational field is generally not warranted, many geodesic formulations that have ignored the angle relation between photon's velocity and gravitational field can hardly produce correct results.

In this section, we'll offer a much simpler, closed-form solution to photon states in a generic gravitational field by using a decomposition method. This method is valid if the Postulation 4 in Section 3 is valid (this section does not depend on the validity of the concept of gravitational mass of photon).

Assume $\mathbf{G}(\mathbf{x})$ is a gravitational field, $\mathbf{V}(\mathbf{x})=\mathrm{d} \mathbf{x} / \mathrm{dt}$ the velocity of a photon at position $\mathbf{x}$, now we need to derive the equations from which the frequency and trajectory of a photon can be computed. Since the speed of a photon is always c, once we have the trajectory of the photon, the tangent on the trajectory gives the direction of the photon.

The discussions in Sections 2 and 3 show that photon has a clever way to react to gravitational field without invoking time dilation, that is, by either changing its frequency or its moving direction, so to cancel out the influence of the gravitational field. As a matter of fact, if the speed of photon in the solution process is constantly c, then time dilation can be ignored and the 4D space-time reduces to 3D space. In both Special Cases I and II, we can solve the trajectories of the photon in 3D space instead of space-time. We must emphasize that space-time is not the golden standard for evaluating a computational method. Fundamental physical principles and satisfaction of observational/experimental data are. Many things can be computed in different ways.

Now, because photon's reaction to the gravitational field depends on the angle between the photon's velocity and the gravitational field, the angle cannot be ignored in general. The following solution to the trajectory of the photon has taken care of this non-isotropic property.

Inspired by the results in Section 2 and 3, we can decompose $\mathbf{G}(\mathbf{x})$ into two components, $\mathbf{G}_{\|}(\mathbf{x})$ and $\mathbf{G}_{\perp}(\mathbf{x})$, such that $\mathbf{G}_{\|}(\mathbf{x})$ is parallel to $\mathbf{V}(\mathbf{x})$, and $\mathbf{G}_{\perp}(\mathbf{x})$ is perpendicular to $\mathbf{V}(\mathbf{x})$, or mathematically,

$$
\mathbf{G}(\mathbf{x})=\mathbf{G}_{\mathbf{I}}(\mathbf{x})+\mathbf{G}_{\perp}(\mathbf{x})
$$

Let $\beta$ be the angle between $\mathbf{G}(\mathbf{x})$ and $\mathbf{V}(\mathbf{x})$, the angle $\beta, \mathbf{G}_{\|}(\mathbf{x})$, and $\mathbf{G}_{\perp}(\mathbf{x})$ can be solved uniquely and explicitly as follows:

$$
\begin{aligned}
& \operatorname{Cos} \beta=(\mathbf{G}(\mathbf{x}) \cdot \mathbf{V}(\mathbf{x})) /(\|\mathbf{G}(\mathbf{x})\|\|\mathbf{V}(\mathbf{x})\|) \\
& \mathbf{G}_{\|}(\mathbf{x})=\mathbf{G}(\mathbf{x}) \cos \beta, \quad \mathbf{G}_{\perp}(\mathbf{x})=\mathbf{G}(\mathbf{x}) \sin \beta
\end{aligned}
$$

Fig. 3(a) illustrates the decomposition of gravitational field $\mathbf{G}(\mathbf{x})$, in which the Bending Radius 
$\mathrm{R}_{\mathrm{B}}(\mathbf{G}(\mathbf{x}))$ and the Bending Center $\mathbf{C}_{\mathrm{B}}(\mathbf{G}(\mathbf{x}))$ are also illustrated, and their computation can be done as follows:

$$
\begin{aligned}
& \mathrm{R}_{\mathrm{B}}(\mathbf{G}(\mathbf{x}))=\mathrm{R}_{\mathrm{B}}\left(\mathbf{G}_{\perp}(\mathbf{x})\right)=\mathrm{H}_{\mathrm{B}}\left(\mathbf{G}_{\perp}(\mathbf{x})\right)=\mathrm{c}^{2} /\left\|\mathbf{G}_{\perp}(\mathbf{x})\right\| \\
& \mathbf{C}_{\mathrm{B}}(\mathbf{G}(\mathbf{x}))=\mathbf{C}_{\mathrm{B}}\left(\mathbf{G}_{\perp}(\mathbf{x})\right)=\mathbf{x}+\mathrm{R}_{\mathrm{B}}\left(\mathbf{G}_{\perp}(\mathbf{x})\right) \mathbf{G}_{\perp}(\mathbf{x}) /\left\|\mathbf{G}_{\perp}(\mathbf{x})\right\|
\end{aligned}
$$

where $\mathrm{H}_{\mathrm{B}}\left(\mathbf{G}_{\perp}(\mathbf{x})\right)$ is used to denote the correct functional relationship between $\mathbf{G}_{\perp}(\mathbf{x})$ and its corresponding Bending Radius $\mathrm{R}_{\mathrm{B}}\left(\mathbf{G}_{\perp}(\mathbf{x})\right)$, just in case Eq. (18)' is improved by future research.

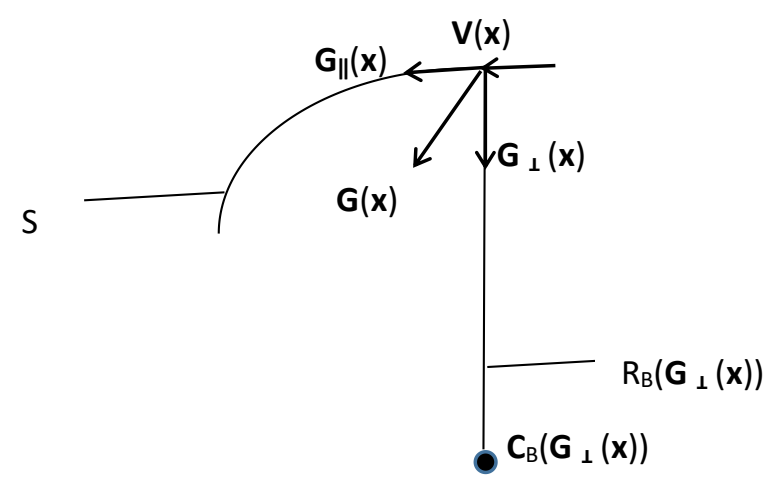

(a)

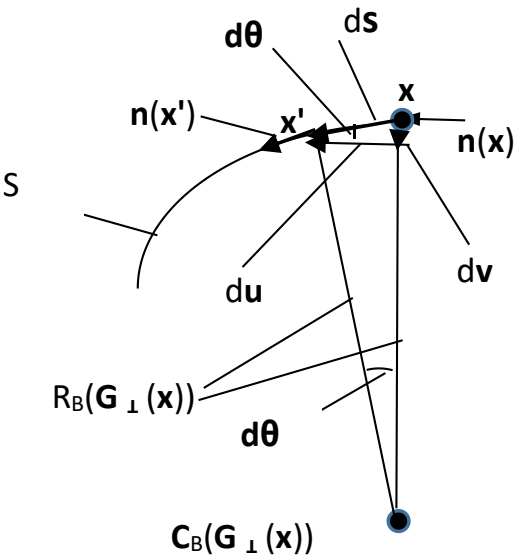

(b)

Fig. 3. Solving photon's states by a decomposition method

The trajectory of the photon can be computed piece-wisely using the method showing in Fig. 3(b), where $\mathrm{d} \theta$ is a small angle departing the Bending Radius $\mathrm{R}_{\mathrm{B}}(\mathbf{G}(\mathbf{x}))$, $d s$ is a small curved length on the Great Photon Circle centered at $\mathbf{C}_{\mathrm{B}}(\mathbf{G}(\mathbf{x}))$ with the Bending Radius $\mathrm{R}_{\mathrm{B}}(\mathbf{G}(\mathbf{x}))$, d $\boldsymbol{u}$ the positional displacement parallel to the Velocity $\mathbf{V}(\mathbf{x})$ corresponding to the displacement of ds, the following relations hold:

$$
\mathrm{ds}=\mathrm{R}_{\mathrm{B}}\left(\mathbf{G}_{\perp}(\mathbf{x})\right) \mathrm{d} \theta, \quad \mathrm{du}=\mathrm{ds} \cos \mathrm{d} \theta
$$

where ds and du are the lengths of $\mathrm{d} \mathbf{s}$ and du respectively.

The next position $\mathbf{x}^{\prime}$ of the photon at position $\mathbf{x}$ is given by $d s$ on the Great Photon Circle centered at $\mathbf{C}_{\mathrm{B}}(\mathbf{G}(\mathbf{x}))$ with the Bending Radius $\mathrm{R}_{\mathrm{B}}(\mathbf{G}(\mathbf{x}))$. Now we give an analytic representation. Let $\mathbf{n}(\mathbf{x})$ be a unit vector of $\mathbf{V}(\mathbf{x})$ such that

$$
\mathbf{V}(\mathbf{x})=\mathrm{c} \mathbf{n}(\mathrm{x})
$$

for any position $\mathbf{x}$. From Fig. 3b, it can be shown that

$$
\begin{aligned}
& \mathrm{d} \mathbf{s}=\mathrm{d} \mathbf{u}+\mathrm{d} \mathbf{v}=\mathrm{du} \mathbf{n}(\mathrm{x})+\mathrm{ds} \operatorname{sind} \theta \mathbf{G}_{\perp}(\mathbf{x}) /\left\|\mathbf{G}_{\perp}(\mathbf{x})\right\| \\
& \mathbf{x}^{\prime}=\mathbf{x}+\mathrm{d} \mathbf{s}
\end{aligned}
$$

where $\mathbf{G}_{\perp}(\mathbf{x}) /\left\|\mathbf{G}_{\perp}(\mathbf{x})\right\|$ is a unit vector along $\mathbf{G}_{\perp}(\mathbf{x})$. The directional vector $\mathbf{n}\left(\mathbf{x}^{\prime}\right)$ of the velocity $\mathbf{V}$ of the photon at $\mathbf{x}^{\prime}$ can be solved from the following equations in linear form:

$$
\begin{aligned}
& \cos (\mathrm{d} \theta)=\mathbf{n}\left(\mathbf{x}^{\prime}\right) \cdot \mathbf{n}(\mathbf{x}) \\
& \mathbf{n}\left(\mathbf{x}^{\prime}\right) \cdot(\mathbf{n}(\mathbf{x}) \times \mathbf{G}(\mathbf{x}))=\mathbf{0} \\
& \left\|\mathbf{n}\left(\mathbf{x}^{\prime}\right)\right\|=\|\mathbf{n}(\mathbf{x})\|=1
\end{aligned}
$$

Finally, the frequency change during this positional change can be computed from (see Eq. (4))

$$
\mathrm{df} / \mathrm{f}=\left\|\mathbf{G}_{\mathbf{l}}(\mathbf{x})\right\| \mathrm{du} / \mathrm{c}^{2},
$$


Note that the above equations hold for every $\mathbf{x}$ regardless the space-time trajectory of the photon, that's because the postulation that photon bends along a local Great Photon Circle defined by a local perpendicular gravitational field is the end result (from any correct solution as defined by Eq. (18)), but not a process. Time $t$ is not even involved. And because in Section 3 we have shown, as long as photon runs on a Great Photon Circle, both special relativity principle and energy conservation principles are automatically satisfied.

Therefore, the above computational process can continue for $\mathbf{x}^{\prime}$ and so on. By continuing the process above, we can compute the trajectory and frequency shift of the photon without even involving time $t$ and the computations are explicit and in closed form. The speed of the photon on the trajectory $\mathrm{S}$ is always $\mathrm{c}$, and the tangent of the trajectory $\mathrm{S}$ at each point is the velocity of the photon at that point. The reason that in this solution time dilation can be ignored is because, in each step, it is automatically satisfied because the solutions are borrowed from Special Cases I and II, which are well established.

Now, the solution in this section does not depend on the correctness of general relativity. It can basically use any correct results of general relativity as in Special Cases I and II. However, The new solution clearly shows that the trajectory of a photon depends not only on the gravitational field, but also its initial velocity (the direction where it is heading initially). This is obvious from intuitions and numerous observations. And the new solution reflects this fact.

In many cases, the gravitational field has a symmetric axis, and the trajectory of the photon is on a plane. In such cases, the equations in Eq. (14)-(20) reduces to 2D equations, further simplifies the computation.

\section{Conclusion}

This article has shown that the isotropic assumption underlies general relativity principles is invalid because photons react to the gravitational field non-isotropically, depending on the angle between the its velocity and the gravitational field. As such, the geodesic method in general relativity cannot be used in general. Further, by assuming photon reacts to gravitational field perpendicular to its velocity through bending its direction with the Bending Radius defined by the local gravitational field, a much simpler, closed form solution is obtained in 3-D space only without even involving time. As such, space-time concept is not necessarily needed in computing photon's states. The only thing that needs observational or experimental confirmation is the computational function $\mathrm{H}_{\mathrm{B}}\left(\mathbf{G}_{\perp}(\mathbf{x})\right)$ of the Bending Radius $\mathrm{R}_{\mathrm{B}}$, which is hypothesized to be given by Eq. (14). Despite this, the whole computational methodology in Section 4 remains sound.

Section 4 shows that the trajectory of photon in a gravitational field near a celestial body depends only on the initial direction of the photon and the mass of the celestial body. Therefore, from the trajectory of the photon, we can actually verify which of the bending radius $\mathrm{G}_{\mathrm{N}} \mathrm{M} / \mathrm{c}^{2}, 2$ $\mathrm{G}_{\mathrm{N}} \mathrm{M} / \mathrm{c}^{2}$, or $3 \mathrm{G}_{\mathrm{N}} \mathrm{M} / \mathrm{c}^{2}$ is correct, and the mass of the celestial body. 


\section{References}

1. Christian G. Bohmer, Introduction to General Relativity and Cosmology, World Scientific, ISSN: 2059-7630 (2017).

2. Tai-Pei Cheng, Relativity, Gravitation, and cosmology, Oxford University Press, ISBN 978-0-19-957363-9, ISBN 978-0-19-9578364-6 (2010).

3. Bernard Schutz, A first course in General Relativity, Cambridge University Press, ISBN 978-0-521-88705-2 (2009).

4. Hugh D. Freedman et al., University Physics, Addison Wesley, ISBN-13: 978-0-321-50121-9, ISBN-10: 0-321-50121-7 (2007).

5. Halliday, David et al., Fundamentals of Physics ( $7^{\text {th }}$ ed.), John Wiley and Sons, ISBN 978-0-471-23231-5 (2005).

6. Randy Harris, Modern Physics ( $2^{\text {nd }}$ ed.), John Wiley and Sons, ISBN-13 978-0-8053-0308-7, ISBN-10 978-0-471-23231-5 (2008).

7. Stephen Hawking, The Universe in a Nutshell, Bantam Books, ISBN 0-553-80202-X (2001).

Note: The first draft of this paper is 0ct. $12^{\text {th }}$, 2019. This version is drafted on Feb. 14 2020. 